\title{
Neocarzinostatin Complex: Chromophore Analogs, Binding Structure and Stabilization Mechanism
}

\author{
Masahiro Hirama \\ Department of Chemistry, Faculty of Science, Tohoku University,
}

\begin{abstract}
Design, synthesis, and chemistry of neocarzinostatin chromophore analogs and their DNA cleaving abilities have been discussed. A Three dimensional solution structure of neocarzinostatin complex explicitly shows how the neocarzinostatin apoprotein binds and prevents the chromophore from decomposing. The suggested binding elements and stabilization mechanisms have been examined by the model studies.
\end{abstract}

\section{Introduction}

Neocarzinostatin (NCS) is a potent antitumor antibiotic isolated from the culture of Streptomyces carzinostaticus by Ishida et al (ref. 1). NCS is a complex composed of an unstable chromophore (1) (ref. 2) and an apoprotein (2) of 11,000 daltons secreted simultaneously (ref. 3). The chromophore (1) is very labile to heat, light, and $\mathrm{pH}$ levels above 6 , but it is stabilized substantially when bound to the apoprotein (2). Thus, the apoprotein (2) serves as a stabilizer and a carrier of the unstable chromophore (1). However, their binding structure and mechanism of stabilization have hitherto been unknown, despite these issues being of major significance in connection with molecular recognition and protein transport. Knowledge concerning all other so-called chromoprotein antibiotics, auromomycin (ref. 4), actinoxanthin (ref. 5),

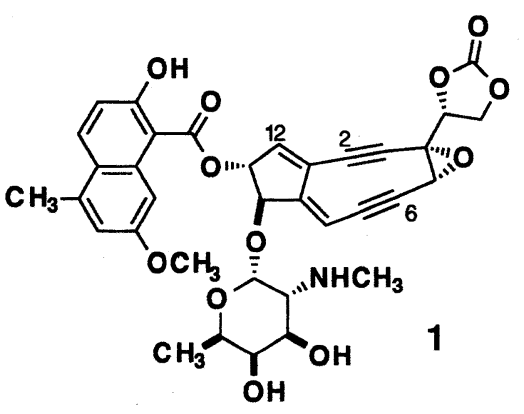

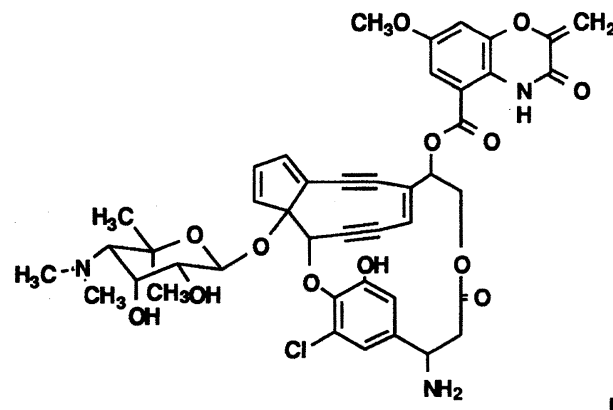

C-1027 Chromophore (3)
Ala-Ala- Pro- Thr- Ala- Thr- Val- Thr- Pro- Ser- Ser- Gly-Leu- Ser- Asp- Gly-Thr- Val- Val- LysVal-Ala- Gly- Ala- Gly- Leu- Gin- Ala- Gly- Thr- Ala- Tyr- Asp- Val- Gly- Gln-Cys- Ala- Trp- Val50 Asp-Thr-Gly- Val- Leu- Ala- Cys- Asp- Pro- Ala- Asn- Phe- Ser- Ser- Val- Thr- Ala- Asp- Ala- Asn-

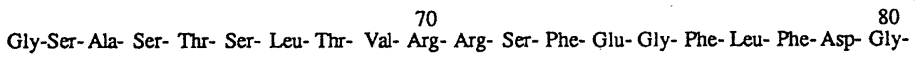
Thr-Arg-Trp- Gly- Thr- Val- Asn-Cys- Thr- Thr- Ala- Ala- Cys- Gln- Val- Gly-Leu- Ser- Asp- Ala110 Ala-Gly-Asp- Gly- Pro- Glu- Gly- Val- Ala- Be- Ser- Phe- Asn

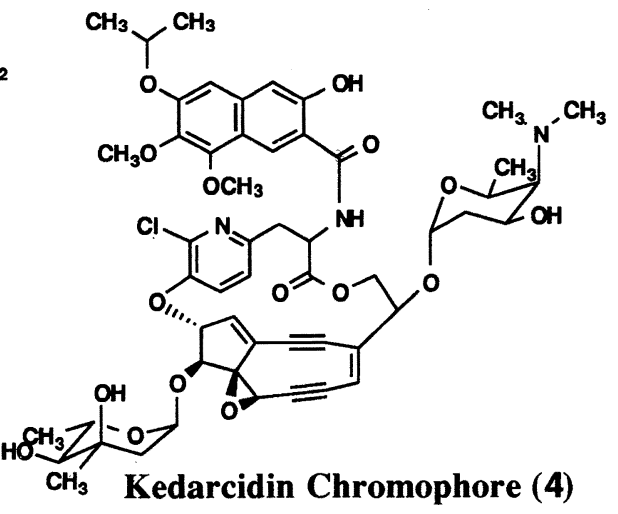


C-1027 (chromophore 3) (ref. 6), and kedarcidin (chromophore 4) (ref. 7) is yet of a similar status quo. Another intriguing issue is the mode of action in connection with those of enediyne antitumor antibiotics (ref. 8, 9). Goldberg and Edo elucidated that 1 is fully responsible for the biological activity of NCS, and that a thiol activates 1 to generate a carbon radical, which abstracts hydrogen from DNA and leads to DNA scission under aerobic conditions (ref. 2). Myers proposed a chemical mechanism for the action of 1: thiol addition to $\mathrm{C} 12$ and a concomitant opening of the epoxide initiates the cascade of reactions that leads to the generation of the dehydroindacene diradical (6) through Masamune-Bergman type cyclization (ref. 8a, 10) of an enynecumulene intermediate (5) (Fig. 1) (ref. 11).

This account reviews our mechanistic and synthetic studies (ref. 8) referring to the following questions and issues:

(1) Is the highly strained 9-membered ring essential for the thiol-triggering aromatization ?

(2) Since there is no precedent for a conjugated enynecumulene system, synthesis of such systems is necessary to understand their chemical behaviors.

(3) Is there any other mode of activation of 1 ?

(4) How does the apoprotein (2) recognize 1 and prevent it from decomposing ?

(5) Is the design and synthesis of new DNA cleaving molecules related to 1 a suitable objective to pursue for attaining low molecular weight, sequence-specific DNA cleavers ?

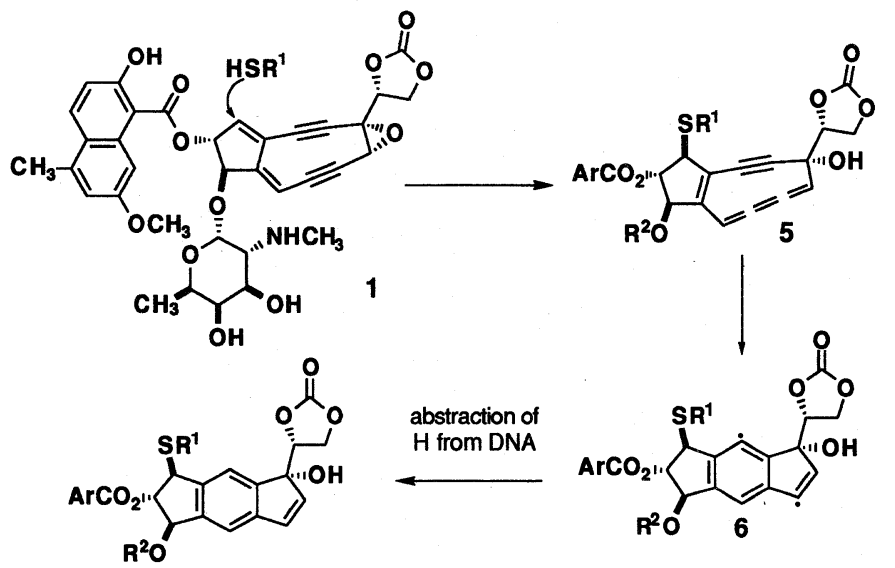

Fig. 1. A proposed mechanism for action of NCS chromophore

\section{Synthesis and Chemical Properties of Acyclic Enynecumulenes}

To address the issues implicit in the first and second questions, we synthesized an acyclic crossconjugated diene-diyne system (7) in a straightforward manner, and treated it with thiol in acetonitrile (ref. 12). Under carefully controlled conditions, we isolated a vinylogous $\mathrm{S}_{\mathrm{N}} 2$ ' reaction product, enynecumulene (8), in moderate yield, although, interestingly, a formally direct substitution product (9) was formed exclusively when a large excess of thiol or DMSO was used as solvent. The ${ }^{13} \mathrm{C}-\mathrm{NMR}$ spectrum strongly supported the cumulene structure for 8 , which was stable at room temperature in the absence of oxygen (ref. 12).
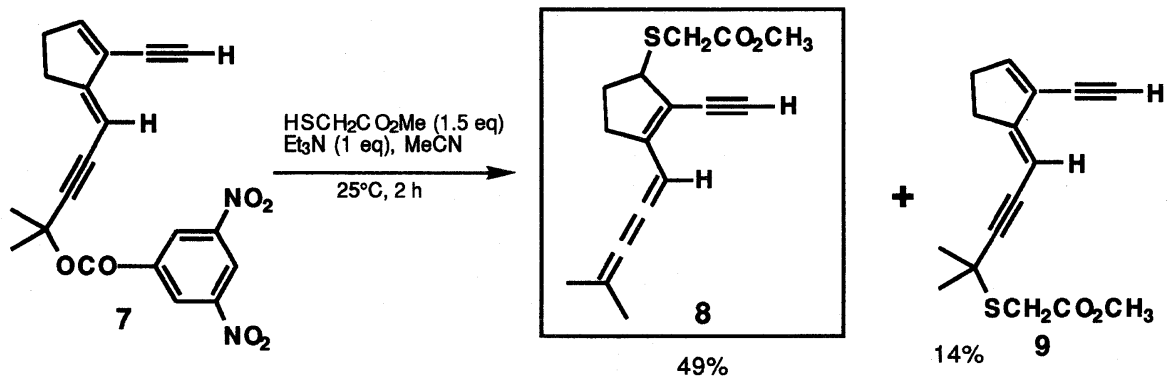

The next question we concerned ourselves with was whether 8 would undergo the MasamuneBergman type cyclization (ref. 10). Heating at $80^{\circ} \mathrm{C}$ in 1,4 -cyclohexadiene produced a styrene derivative 
(10). When the reaction was carried out in cyclohexadiene-d8, two deuteriums were incorporated at the relevant positions, which clearly indicates that such an enynecumulene system has an ability to undergo the cycloaromatization leading to the generation of $\sigma, \sigma$-diradicals. The first-order disappearance rate of 8 is approximately 30 times slower than that of the related enynallene (12) (ref. 13). In addition, benzocyclobutane (11) formation turned out to be another major reaction pathway in this conformationally flexible system (ref. 12). Recently Brückner has supplemented another mechanistic rationale for the formation of $\mathbf{1 1}$ (ref. 14).

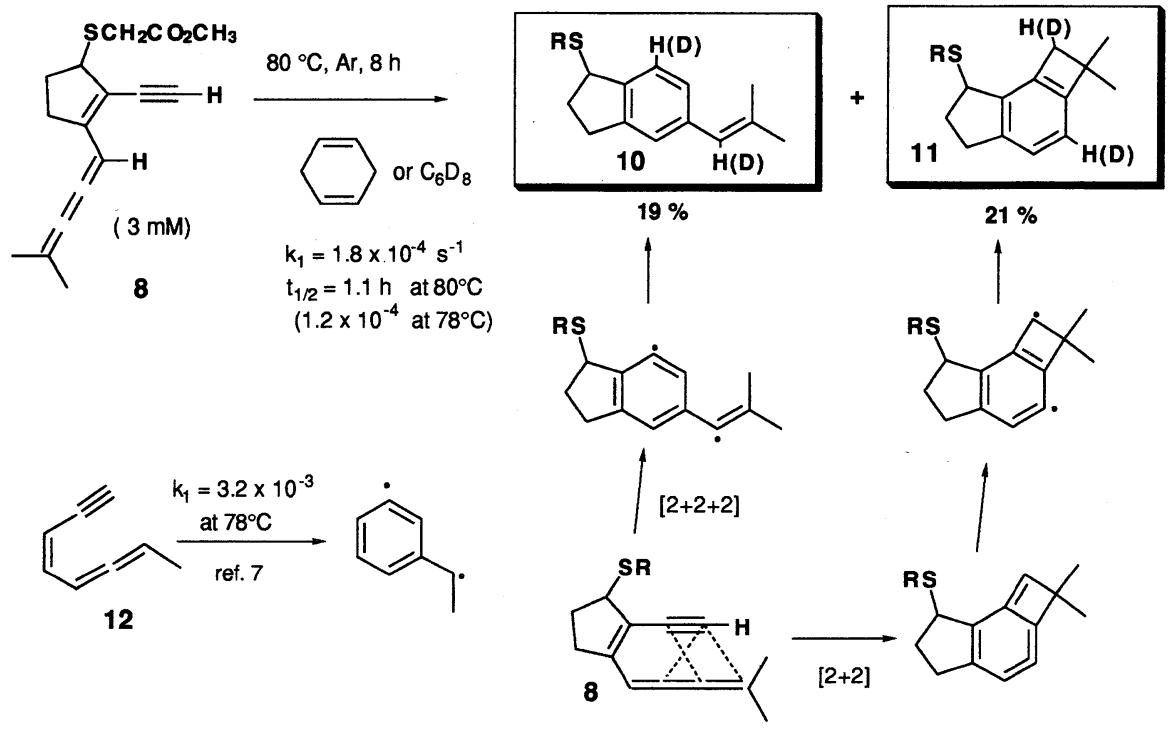

\section{Synthesis, Cycloaromatization, DNA Cleaving and Biological Activities of 10- Membered Ring Analogs}

\subsection{Synthesis}

In connection with the first question, a cyclic, less strained 10-membered analog is an another intriguing system. Noteworthy in the syntheses of the 10 -membered ring analogs (15-17) is the palladium-mediated effective internal cyclization of vinyl bromide-alkynyl stannane (14) (ref. 15). Attempts of direct cyclization of 13 without the stannyl group failed, and instead resulted in the formation of dimeric oxidative coupling products. The final oxidation and concomitant dehydration proceeded smoothly by Swern oxidation (ref. 16, 17).
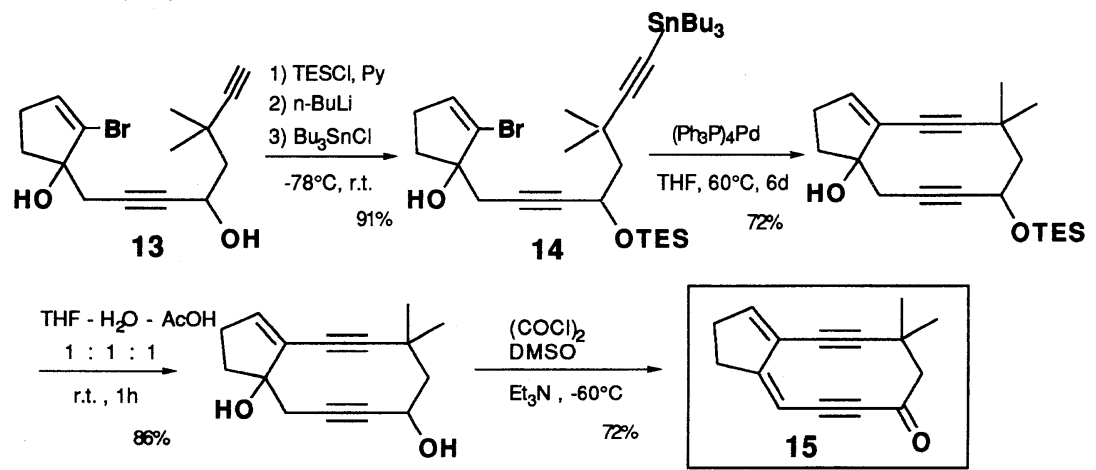

\subsection{Radical Induced Cycloaromatization}

It was demonstrated that analogs (15-17) can undergo the thiol initiated aromatization at room temperature as well as thiyl or hydroperoxy radical induced cycloaromatization (ref. 16, 17). The less 
strained dienediyne system seems to have a marked tendency to react with radical species at $\mathrm{C} 13$ leading to a delocalized radical intermediate such as 19. Smooth formation of an unstable epoxide 18 from 16 with molecular oxygen was rationalized on the basis of formation of the radical intermediate 19 (ref.18).

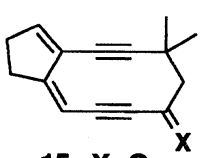

$15, X=0$ $16, X=H, O A C$
$\mathrm{HSCH}_{2} \mathrm{CO}_{2} \mathrm{CH}_{3}(3 \mathrm{eq})$ $\mathrm{NEt}_{3}(1 \mathrm{eq}), \mathrm{Ar}$

EtOH, $25^{\circ} \mathrm{C}, 10 \mathrm{~min}$<smiles>COC(=O)CSC1CCc2cc3c(cc21)C(C)(C)CC(=O)C3</smiles>

$54 \%$

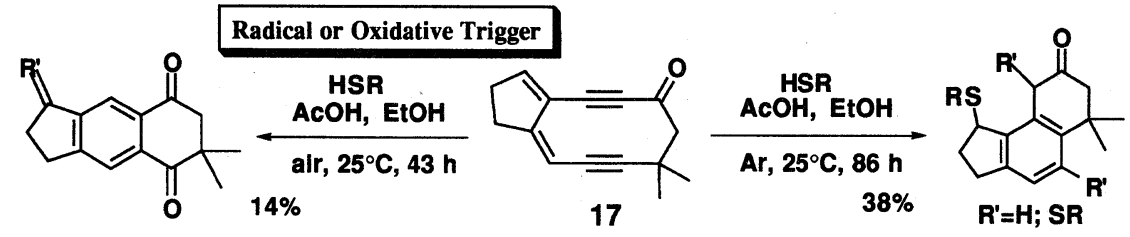

R'=H,OH; H,SR; O

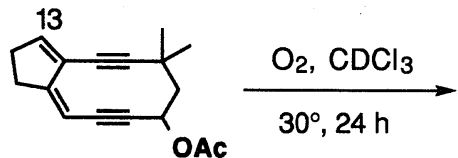

16

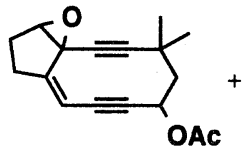

18

( $6: 1)$<smiles>CC(=O)OC1CC(C)(C)c2c(cc3c(c2O)C(=O)CC3)C1=O</smiles>

)

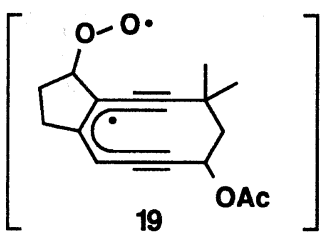

\subsection{New Initiation Modes of Cycloaromatization of NCS Chromophore}

\subsubsection{Radical Triggering Pathway}

The radical induced aromatization demonstrated in the reaction of 10-membered analogs (15-17) has also been observed in the aromatization of NCS chromophore (1). Formation of ketone 20 (ca. $10 \%$ yield) was confirmed by decomposition of 1 in methanolic acetic acid in the presence of air and methyl thioglycolate at $-30^{\circ} \mathrm{C}$ in the dark (ref. 19). This experiment suggested that the aromatization of 1 triggered by a hydroperoxy radical formed from thiol and molecular oxygen would become important under the physiological conditions when nucleophilic thiols such as glutathione are insufficient.

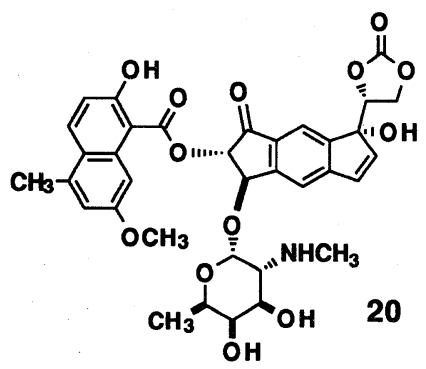

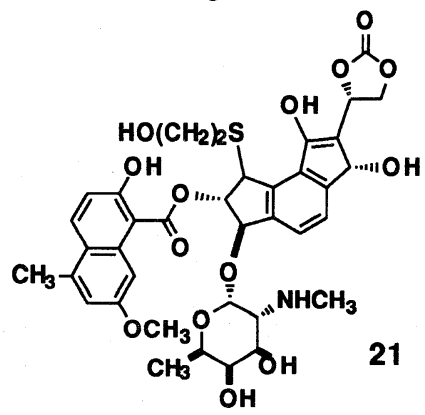

\subsubsection{New Mode of Triggering}

On the other hand, we suggested an alternative activation pathway of 1 (Fig. 2) based on the model studies using 17 (ref. 17). Saito et al. recently found that aromatization product 21 related to this new activation mode was produced as a major product when 1 was incubated with 2mercaptoethanol in the presence of apoprotein (2) in aqueous solution, although it is likely to be an ionic pathway and therefore will not partake in the DNA cleavage mechanism. 


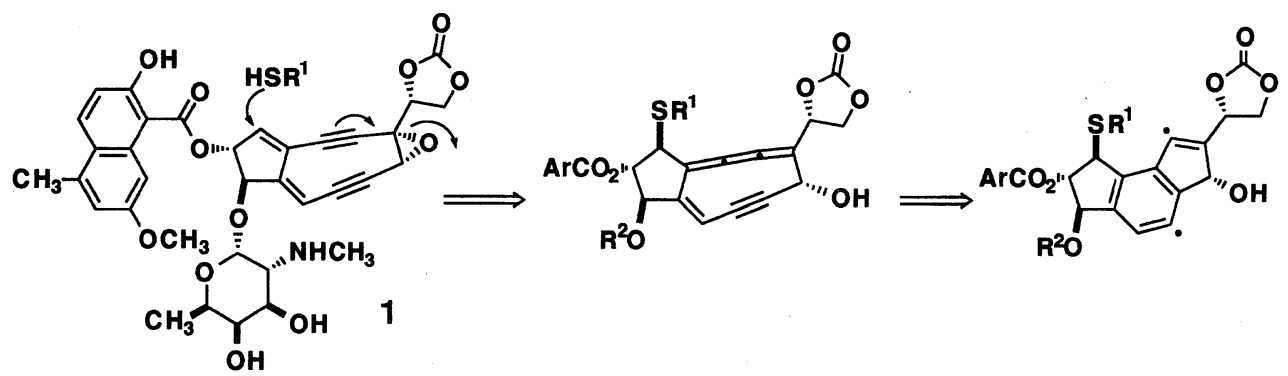

Fig. 2. An alternative activation pathway of NCS chromophore

\subsection{Second Generation of NCS Chromophore Analogs}

Analogs 15 and 16 did not exhibit DNA cutting or cytotoxic activities, possibly because they lack a hydrophilic or DNA binding group. Therefore, we designed the second generation models (2225) to improve these activities (ref. 21-23). Our basic strategy for designing new DNA cleaving molecules was to combine the DNA cleaving unit with some DNA binding group.

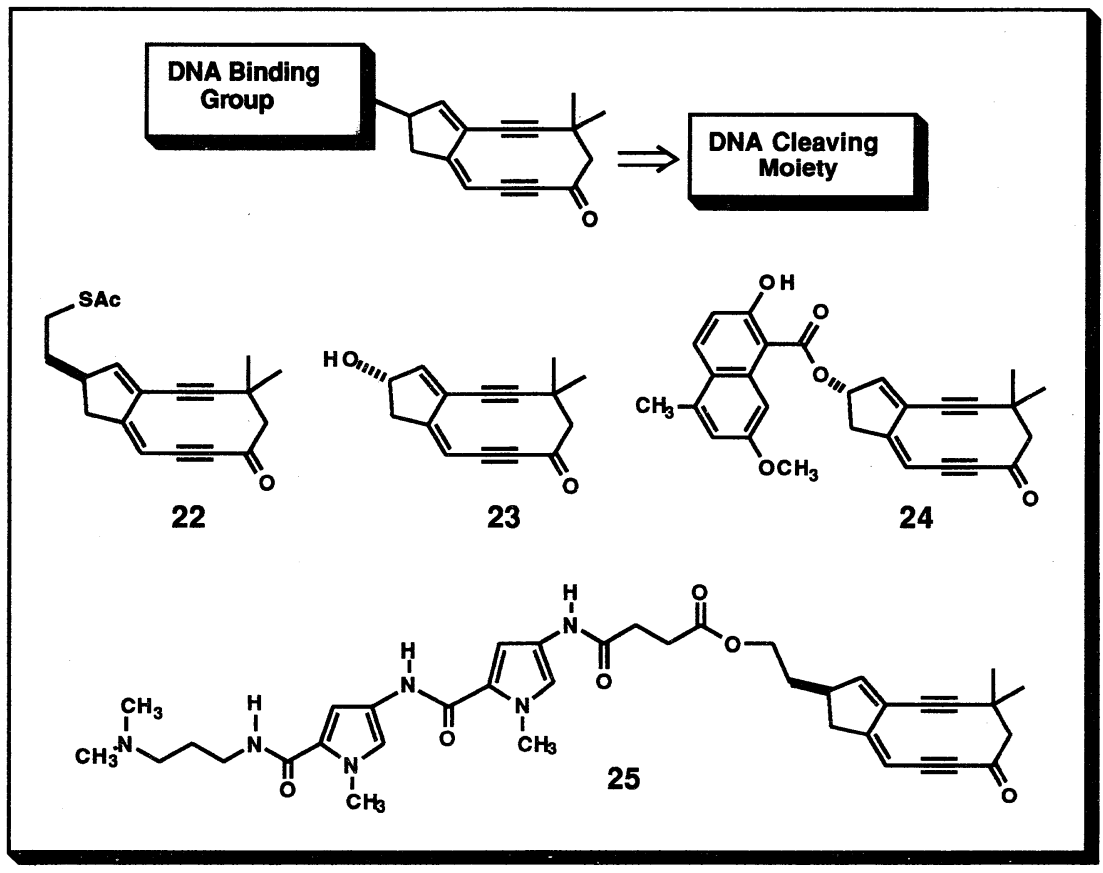

\subsubsection{A New Model Equipped with an Intramolecular Trigger}

First, we synthesized a new model (22) equipped with an intramolecular nucleophile, a design idea contrived from the related potent antitumor antibiotics, calicheamicin and esperamicin (ref. 8a, 9a). Methanolysis using potassium carbonate in the presence of 1,4-cyclohexadiene at room temperature was complete within 30 minutes in an argon atmosphere to give the reductive cycloaromatization product through the intramolecular addition of thiolate (ref. 21). Deuterium incorporation was observed at C-2 in the presence of cyclohexadiene- $\mathrm{d}_{8}$. This internal trigger model (22) showed an appreciable activity toward Micrococcus luteus without addition of external thiol.

\subsubsection{New Functional Analogs}

The naphthoate conjugate $\mathbf{2 4}$ is of interest, particularly in connection with two issues: Firstly, it would exhibit a T-selective DNA cleavage similar to that of 1 (ref. $2 a$ ). If so, it should provide an 
evidence that the naphthoate moiety is responsible for T-specific DNA scission by 1 through its specific recognition of T residue by intercalation (ref. 24). Secondly, the NCS apoprotein (2) would serve as a carrier of this lipophilic molecule (24), since our recent NMR studies of the three dimensional structure of the NCS complex (vide infra) have shown that the naphthoate moiety is responsible for the specific binding of the chromophore (ref. 25).

The enantiomerically pure alcohol (23) and naphthoate conjugate (24) were then synthesized. Their thiol triggered cycloaromatization which proceeded smoothly at room temperature in ethanol in the presence of 1 equivalent of triethylamine (ref. 22).

The alcohol (23) clearly showed antibiotic and cytotoxic activities against $B$. licheniformis and K562 leukemia cell, respectively. On the other hand, the activities of naphthoate (24) alone were quite low, but in the presence of 2 , the mixture exhibited the distinctive activities. This is likely to result from the complex formation and transport function of 2. Similar results were again observed in the DNA cleavage experiments. The naphthoate 24 showed some sequence selective DNA cleavage activity, namely cutting G of 5'-GGT and T of 5'-GCT, but only in the presence of 2 (ref. 22, 8b). Since this T selectivity is characteristic of the selectivity of 1 , it implies that the naphthoate moiety should play an important role for the site selective DNA cleavage of 1 . On the other hand, remarkable G specificity was found for alcohol 23. This was surprising because 23 cannot be an intercalator nor does it possess an apparent DNA binding moiety. Recent detailed DNA cleavage experiments suggest that $\mathrm{G}$ specificity for $\mathbf{2 3}$ might arise from its chemical reactivity (alkylation mechanism) rather than the molecular recognition of a guanine base followed by DNA cutting via a radical mechanism (ref. 26).

\subsubsection{A New Hybrid Molecule Containing a Minor Groove Binder}

The antibiotic netropsin is known to be a strong minor groove binder to the AT-rich regions of DNA through hydrogen bonding and van der Waals interactions. So, we anticipated that the netropsin conjugate (25) would function as an effective AT-specific DNA cleaver. Synthesis of optically pure 25 has been achieved (ref. 23). Because 25 possesses an internal amine, it undergoes the thiol-triggering cycloaromatization quickly without addition of an external amine as expected. We compared the DNA cleaving abilities of synthetic molecules 22, 23, and 25 on supercoiled pBR322 DNA. Agarose gel electrophoresis clearly showed that netropsin conjugate (25) cleaved supercoiled DNA-Form I to give a nicked circular Form II and then further to form a linear Form III in a highly efficient manner (ref. 23).

\section{Apoprotein-Chromophore Binding Structure and Stabilization Interactions}

\subsection{Solution Structure and Binding Elements}

Our recent study of three-dimensional solution structure of the NCS complex utilizing 2D NMR and distance geometry calculations revealed the elements of the specific binding and the stabilization interactions between unstable 1 and 2 (fig. 3) (ref. 25). This is the first time that the chromophorebinding structure has been determined in a member of the chromoprotein antibiotic family. A hydrophobic pocket with approximate dimensions of $12 \times 9 \times 9 \AA$ is the binding site of 1 . Apoprotein (2) accepts the naphthoate moiety of 1 deeply into this pocket. The C5"-C7" site of the naphthoate is in proximity to the bottom of the pocket where the main chains of three strands (Val34-Gly35-Gln36, Leu97-Gly96-Val95, and Gly107-Val108) are exposed. The carbocyclic core is located on the Cys37Cys47 disulfide bond, and the aminosugar and the carbonate moieties are facing outwards. There seems to be no aromatic stacking interaction with the naphthalene ring, such as that predicted by computer modeling (ref. 25a). Instead, several other principal interactions are indicated. The oxygen atoms of C7"-O and C2"-O of the naphthoate of 1 are close enough to OH of Ser98 and NH of Trp39, respectively, to produce a hydrogen bond. The C5"-methyl is approximately in van der Waals contact with the $\beta$-methylene of Gln94. The upfield shift of the $\mathrm{C}^{2}$-- $\mathrm{OCH}_{3}$ resonance by $1 \mathrm{ppm}$ upon binding is most likely due to the diamagnetic anisotropy of the nearby aromatic ring of Phe52, which supports a possible $\mathrm{CH}-\pi$ interaction (ref. 27 ).

On the other hand, the C2-C3 triple bond of the core is just above the sulfur atom of Cys47, and is covered perpendicularly by the aromatic ring of Phe 52. The aromatic ring of Phe78 is surrounding the other side of the core, the C6-C7 triple bond. It can be concluded therefore that Phe78 and Phe52 as well as Cys37-Cys47 disulfide bond must be of central importance for holding the carbocyclic core 


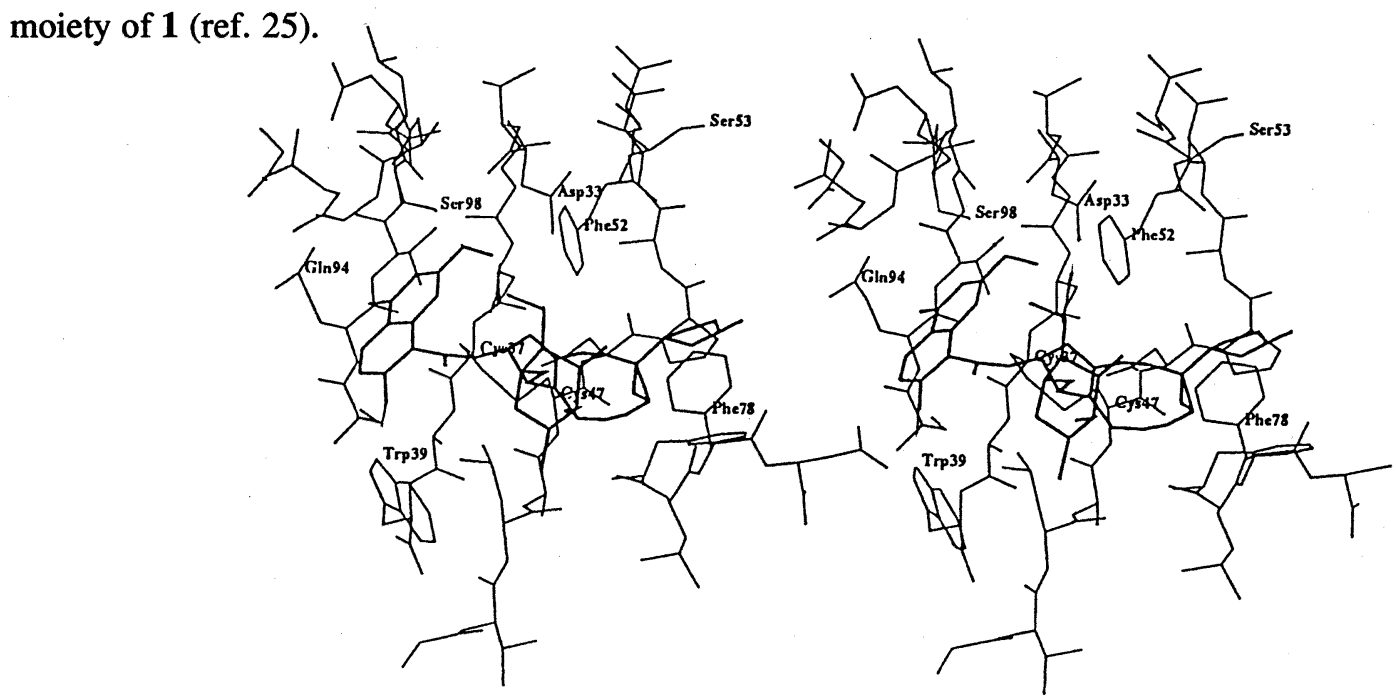

Fig. 3. Binding structure of NCS complex

Recent X-ray crystallographic study (ref. 28) of the NCS holoprotein structure showed a different conformation of Phe 78 aromatic ring from that of our solution structure (ref. 25c). So, we have carried out further analyses combining the crystallographic data of the apoprotein (2) (ref. 29) and the NMR NOEs (ref. 25) observed between 2 and 1 (ref. 30). It suggests that the aromatic moiety of Phe78 changes significantly in its conformation upon binding of 1 and that this moiety in the holoprotein is still rather flexible in solution. We conclude that Phe78 in the crystal structure of the holoprotein exists as one of the stable conformers, whereas Phe78 is dynamically undergoing a multiple of conformations in the solution. Though the overall shape of the binding pocket of 2 is similar to the related chromoproteins, auromomycin (ref. 4) and actinoxanthin (ref. 5), the side chains of the residues along the binding pocket are very different in size and functionality with exception of the Cys37-Cys47 disulfide. Since NCS-chromophore (1) is a poor ligand for auromomycin apoprotein (ref. 4), it is very likely that the residues described above are crucial for chromophore binding specificity. In particular, Phe 78 and Phe 52 are not conserved in other chromoprotein antibiotics. The interactions between the core acetylenic bonds and the Phe 52 and Phe 78, in a similar manner to an aromatic edge-to-face interaction, should be important for the specific binding of the core moiety of 1 in the holoprotein. Since Phe 78 has multiple conformers covering the core, it should play an important role for release of the chromophore (1) and for the protection of 1 in the holoprotein (ref. 25, 30).

\subsection{Confirmation and Evaluation of Binding Elements}

\subsubsection{Synthesis of Ligands}

The binding structure is indicative of the idea that the naphthoate moiety of 1 must be essential for its specific and strong binding to 2 , and that the several interactions between the functional groups on the naphthoate ring and the side chains of residues (Fig. 4) appear to be responsible for such an important role (ref. 25). To confirm the importance of the naphthoate moiety of 1 for the specific binding, we synthesized simple analogs (26-31) in Fig. 5 and carried out their binding experiments to the apoprotein (2) (ref. 31). On this occasion, we needed an efficient synthetic way to access the naphthoic acid (32). The reported syntheses are elegant but too laborious for our practical purposes. So, we have developed a simple and versatile route to 32 . This synthetic strategy is useful to synthesize the related 2-hydroxy1-naphthoic acid derivatives (ref. 31 ).

Then, we tried to synthesize glycoside analogues 30 and 31 with the natural $\alpha$-stereochemistry from 26 and 27 by developing an efficient means of coupling these alcohols with an appropriate $N$ methyl- $D$-fucosamine donor directly without protection of the $N$-methylamino group. We envisioned that the neighboring group participation of the $N$-methylamino group of such a donor in the glycosidation can be inhibited in the presence of excess strong acids or silylating agents through in situ formation of 


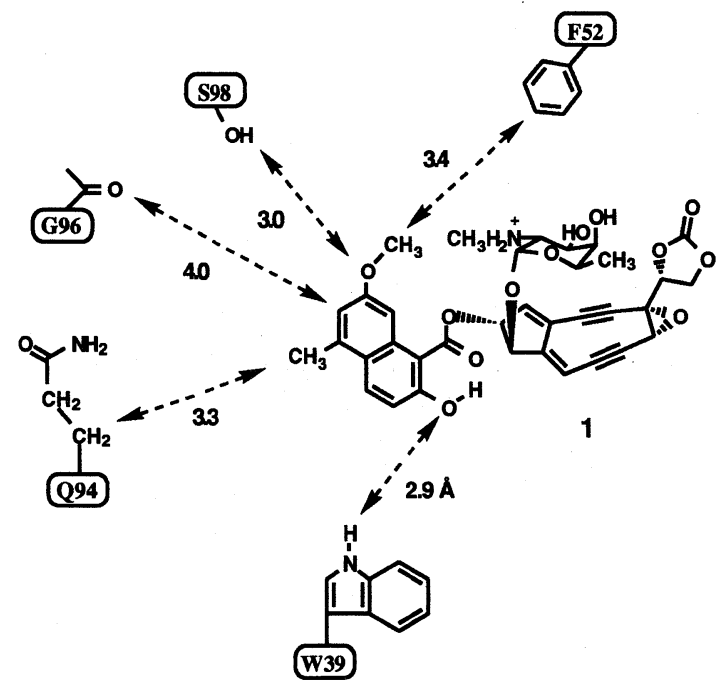

Fig. 4. Binding interactions between the naphthoate moiety of 1 and the amino acid residues of 2.
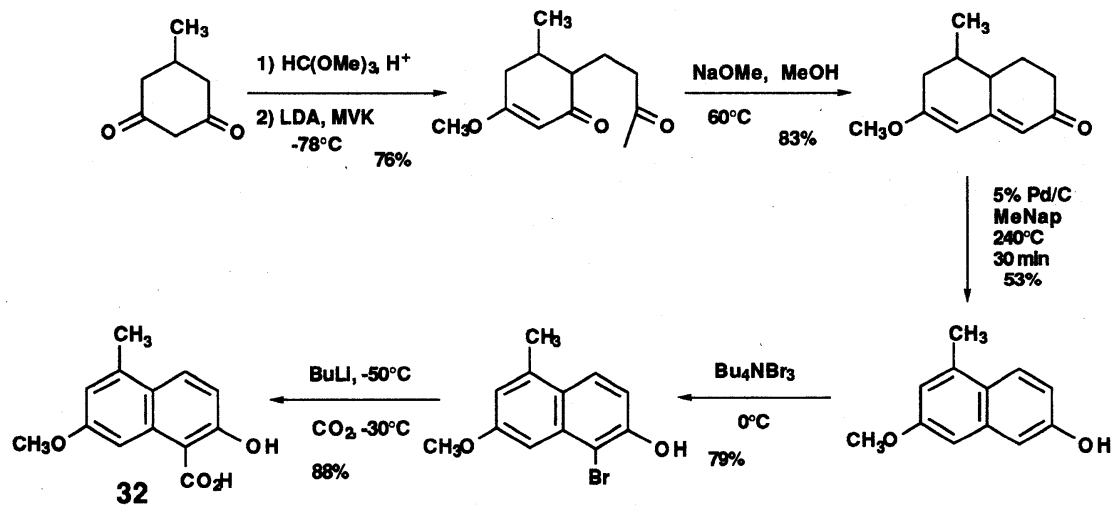

32

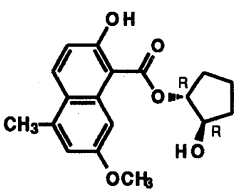

26
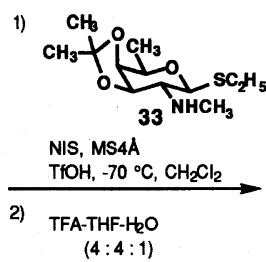

$23 \%$
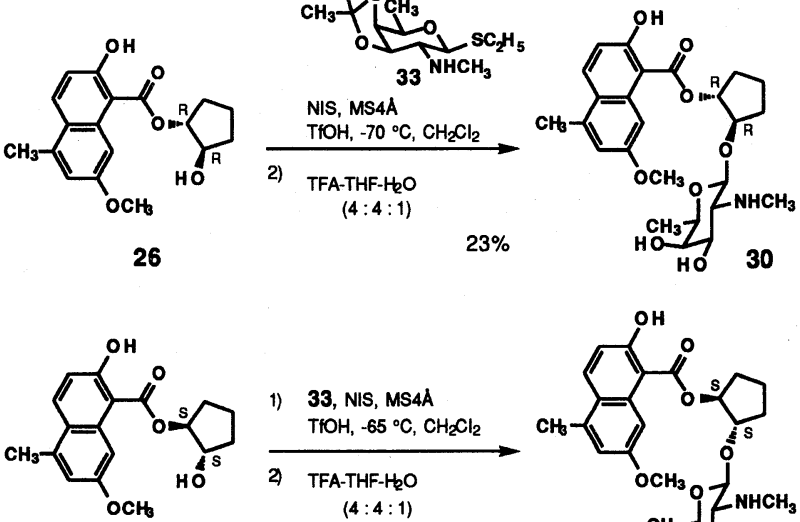

$49 \%$

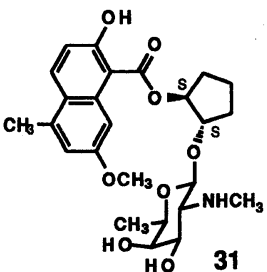

the ammonium salt. Activation of thioglycoside 33 with NIS/TfOH or NIS/TESOTf in $\mathrm{CH}_{2} \mathrm{Cl}_{2}$ (ref. 32) accomplished the glycosidation in reasonable yields, although the reaction lacked stereocontrol. The 
$\beta$-glycosides 30 and 31 were obtained stereoselectively from 26 and 27, respectively (ref. $31 \mathrm{~b}$ ). A stereoselective $\alpha$-glycosidation methodology for 33 and other relevant donors is currently under investigation.

\subsubsection{Association Constant}

The binding constants of the model compounds (26-31), obtained by fluorescence quenching titration, supported the above idea that the naphthoate moiety is a necessary design feature for sustaining this binding (Fig. 5) (ref. 25). While the bindings of the naphthoates 26 and 27 are still 200-fold weaker than the natural chromophore (1), their binding energies reach to approximately $64 \%$ of the total binding energy of 1 . The binding affinity of the demethylated analog 29 is approximately one third of that of 26, supporting the van der Waals contact between $\mathrm{C}_{5}-\mathrm{CH}_{3}$ and the $\beta$-methylene of Gln94. While the demethylated diol 28 also showed a lower affinity than 26,28 should be able to retain the hydrogen-bonding with Ser98. So, the $K a$ difference between 28 and 26 appears to correspond to the loss of a $\mathrm{CH}-\pi$ interaction between $\mathrm{C} 7-\mathrm{OCH}_{3}$ and the nearby aromatic ring of Phe 52 . This $\mathrm{CH}-\pi$ interaction could be then estimated approximately as $-2.8 \mathrm{KJ} / \mathrm{mol}$ (ref. $31 \mathrm{~b}$ ). The $K a$ of the $\beta$-glycoside 30 is very close to that of $\mathbf{2 6}$, suggesting that the wrong $\beta$-stereochemistry has only negligible effects on the binding. Approximately 10-fold higher affinity of $\mathbf{3 1}$ than 27 and $\mathbf{3 0}$ is interesting. These results would be discussed after the corresponding $\alpha$-glycosides become available in our hand.

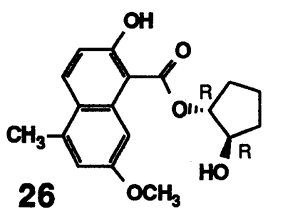

$\mathrm{Ka}=1.3 \times 10^{4} \mathrm{M}^{-1}$

$\Delta \mathrm{G}=-23.5 \mathrm{KJ} / \mathrm{mol}$

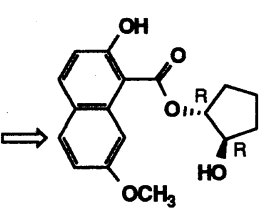

29

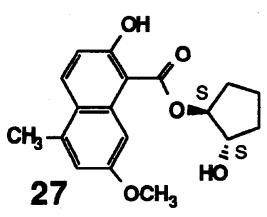

$\mathrm{Ka}=1.5 \times 10^{4}$

$\Delta \mathrm{G}=-23.8$

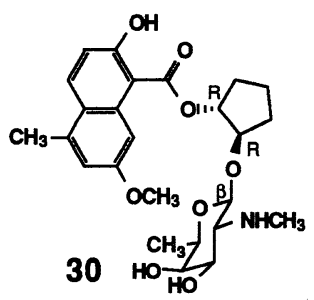

$\mathrm{Ka}=2.0 \times 10^{4}$

$\Delta \mathrm{G}=-24.5$

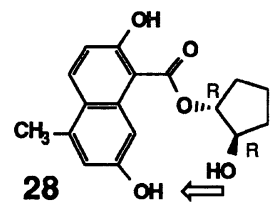

$\mathrm{Ka}=4.2 \times 10^{3}$

$\Delta \mathrm{G}=-20.7$

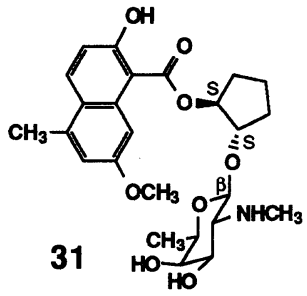

$\mathrm{Ka}=1.6 \times 10^{5}$

$\Delta \mathrm{G}=-29.7$

NCS Chromophore : $\mathrm{Ka} \mathrm{a}^{\prime} \geq 3 \times 10^{6} \mathrm{M}^{-1}$ in $5 \% \mathrm{MeOH} / 0.1 \mathrm{M}$ acetate buffer (pH 4.0)

$\Delta \mathrm{G} \leq-37 \mathrm{KJ} / \mathrm{mol}$

Fig. 5. Binding constants of simple model compounds to apoprotein (2)

\subsection{Stabilization Interactions}

\subsubsection{Chemical Stability}

The conformation of bound chromophore (1) in the complex showed some interesting features. The sugar portion hangs over the 5-membered ring of the core with the hydrophobic side down, thus satisfying the exo-anomeric effect (Fig. 6). The methylamino group is forced to come closer to C12 $(4.3 \AA)$ partly due to salt bridge with Asp33 compared with the conformation of uncomplexed 1 (5.4 $\AA$ ). The naphthoate stays away from the core to fit into the hydrophobic pocket of 2 , thus allowing the core 
to lie on the disulfide bridge, while in the stable conformations of free form it lies just below the core to become more compact. The side chains of Phe52, Phe78, and Asp33, and the Asp99-Asn103 loop cover the reactive center $\mathrm{C} 12$ as well as the acetylenic bonds. These steric hindrances in $\mathrm{C} 12$ and the acetylenic carbons should be a major stabilizing factor for 1 (ref. 25, 30).
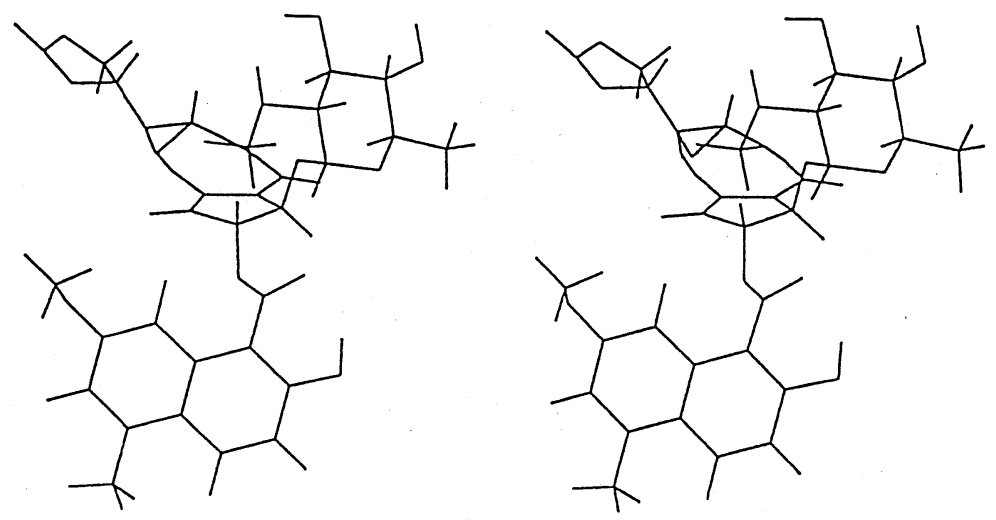

Fig. 6. The apoprotein-bound conformation of the chromophore (1)

\subsubsection{Thermal Stability}

The naphthoate moiety was found to be shifted away from the core so as to fit into the pocket of 2, so that the plane of the carbocyclic core was located on the Cys37-Cys47 disulfide bridge. Their distance was close enough (about $3.4 \AA$ ) to have an orbital interaction. Sulfur atom of Cys37 was situated at $3.4 \AA$ from the center of $\mathrm{C} 12-\mathrm{C} 1$ double bond and the sulfur atom of Cys 47 was near to the acetylenic carbon $\mathrm{C} 2$. The disulfide bond was parallel to the core plane. Therefore, HOMO (the dienediyne $\pi$-system)-LUMO (vacant sulfur d orbital) or LUMO (dienediyne)-HOMO (sulfur lone pair) interaction would stabilize the core $\pi$-system. Since this disulfide bridge is conserved in all chromoprotein antibiotics, such an interaction with the strained enediyne chromophore may be common in this family (ref. 25, 30).

\subsubsection{Photochemical Stability}

The stability of 1 is significantly reduced under the ambient light. However, 2 protects 1 against photo-decomposition (ref. 2). Recently we found that the central pathway of photochemical decomposition of the analog (16) proved to be a fragmentation reaction of a naphthoate ester, a reaction which is anologous to a Norrish Type II split, leading to the formation of an unstable fulvene derivative (34) and the naphthoic acid (32), which implied the photodecomposition mechanism occuring in 1 (ref. 33).

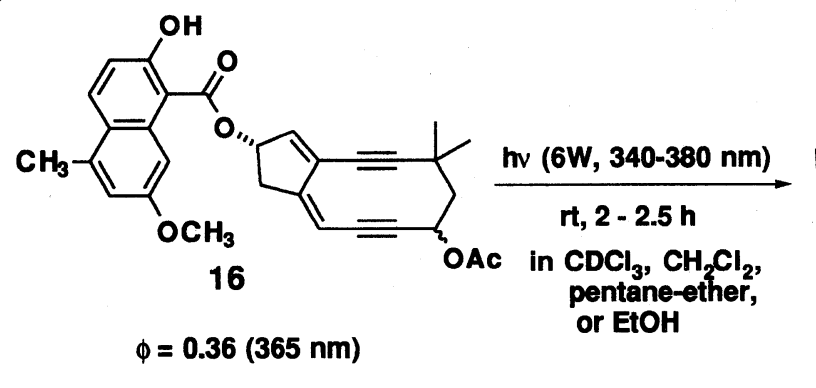

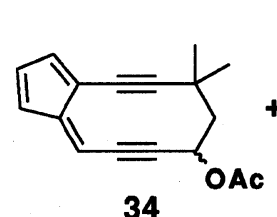

$0-25 \%$<smiles>COc1cc(C)c2cc(O)c(C(=O)O)c(O)c2c1</smiles>

$19-24 \%$

In particular, the naphthoate carbonyl of 1 should be excited by light and would thus undergo photoreactions such as Norrish Type II fragmentation leading to a very labile enol-glycoside (35). Such processes can compete with the reversible abstraction of the hydroxyl-H to form the enol (36). Two stabilizing factors to prevent 1 from undergoing photo-decomposition were indicated by the threedimensional structure of the NCS complex (Fig. 3) (ref. 25). First, several binding interactions between 
the naphthoate and the binding-site residues could quench the excited state. Second, in the bound chromophore the naphthol proton is fixed very closely $(2.0 \AA)$ to the ester carbonyl (Fig. 6). There must be a strong intramolecular hydrogen bonding in the bound conformation, which should promote the hydroxyl-H 1,5 shift leading to a photo-enol equilibrium and subsequent quenching of the excited state (Fig. 7).

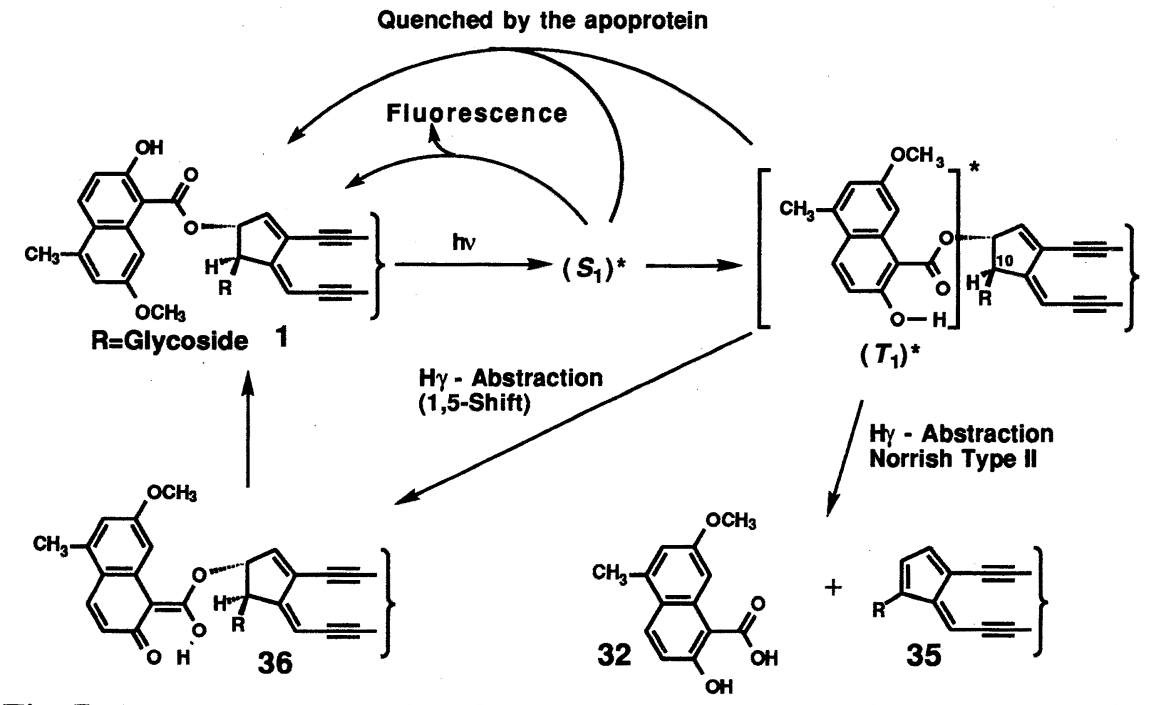

Fig. 7. A proposed mechanism for photo-decomposition of chromophore (1)

\section{Conclusion}

Rational design of new DNA cleaving molecules inspired by the NCS chromophore (1) has provided us with the structural and mechanistic probes for chemical functions of the carbocyclic dienediyne core and substituents of 1 . A combination of model studies using synthetic analogs with NMR studies on three dimensional structure of the neocarzinostatin complex enabled us to understand the ligand-protein interactions (ref. 8). We anticipate that other members of this chromoprotein family such as $\mathrm{C}-1027$ and kedarcidin will be studied using similar approaches.

\section{Acknowledgment}

I wish to thank my talented and enthusiastic coworkers, Dr. K. Fujiwara, Dr. T. Tanaka, Dr. T. Suzuki, M. Tokuda, T. Gomibuchi, K. Takahashi, and express my sincere thanks to Prof. Y. Sugiura (Kyoto University; DNA cleavage), Dr. M. Ishiguro and Dr. S. Imajo (Suntory Co. Ltd.; DGEOM calculation), K. Fujita (JEOL Co. Ltd.; MolSkop® calculation), and Dr. H. Komatsu (POLA Co. Ltd.; biological activity) for their great help and fruitful collaborations. Thanks are also due to Professors N. Ishida (Emeritus Prof. of Tohoku Univ.), M. Mizugaki (Tohoku Univ.), and K. Edo (Fukushima Medical College) for their valuable discussions. Financial support for the work described herein is greatfully acknowledged: Ministry of Education, Science and Culture, Japan, Japanes Society for Promotion of Science, Mitsubishi Science Foundation, CIBA GEIGY Foundation, Shorai Science Foundation, TERUMO Life Science Foundation, Takeda Science Foundation, and Fujisawa Medicinal Resources Foundation.

\section{References}

(1) Ishida, N.; Miyazaki, K.; Kumagai, K.; Rikimaru, M. J. Antibiot. 1965, 18, 68.

(2) (a) Goldberg, I. H. Acc. Chem. Res. 1991, 24, 191. (b) Edo, K.; Mizugaki, M.; Koide, Y.; Seto, H.; Furihata, K.; Otake, N.; Ishida, N. Tetrahedron Lett. 1985, $26,331$.

(3) Kuromizu, K.; Tsunasawa, S.; Maeda, H.; Abe, O.; Sakiyama, F. Arch. Biochem. Biophys. 1986, 246, 199. Kappen, L. S.; Goldberg. I. H. Biochemistry 1980, 19, 4786. 
(4) Roey, P. V.; Beerman, T. A. Proc. Natl. Acad. Sci. USA 1989, 86, 6587.

(5) Pletnev, V. Z.; Kuzin, A. P.; Trakhanov, S. D.; Kostetsky, P. V. Biopolymers 1982, 21 , 287.

(6) Minami, Y.; Yoshida, K.; Azuma, R.; Saeki, M.; Otani, T. Tetrahedron Lett. 1993, 34, 2633. Yoshida, K.; Minami, Y.; Azuma, R.; Saeki, M.; Otani, T. Tetrahedron Lett. 1993, 34, 2637.

(7) Leet, J. E.; Schroeder, D. R.; Langley, D. R.; Colson, K. L.; Huang, S.; Klohr, S. E.; Lee, M.S.; Golik, J.; Hofstead, S. J.; Doyle, T. W.; Matson, J. A. J. Am. Chem. Soc. 1993, 115, 8432.

(8) (a) Fujiwara, K.; Hirama, M. Gendai Kagaku (Chemistry Today) 1990, 7, 14. (b) Hirama, M. J. Synth. Org. Chem. Jpn. 1991, 49, 1032. (c) Hirama, M. Kagaku 1992, 47, 162. (d) Hirama, M. in Recent Progress in the Chemical Synthesis of Antbiotics and Related Microbial Products, Vol. 2; Lukacs, G. Ed; Springer-Verlag: Berlin, 1993; pp 293. (e) Hirama, M. in Antibiotics and Antiviral Compounds, Chemical Synthesis and Modification; Krohn, K.; Kirst, H. A.; Maag. H. Ed.; VCH: Weinheim, 1993, pp 289. (f) Hirama, M.; Tanaka, T. Pure and Appl. Chem. 1994, 66, 791.

(9) (a) Nicolaou, K. C.; Dai, W.-M. Angew. Chem. Int. Ed. Engl., 1991, 30, 1387. (b) Skrydstrup, T.; Ulibarri, G.; Audrain, H.; Grierson, D. S. in Recent Progress in the Chemical Synthesis of Antibiotics and Related Microbial Products, vol. 2; Lukacs, G. Ed; Springer-Verlag: Berlin, 1993; pp 213.

(10) Darby, N.; Kim, C. U.; Salaün, J. A.; Shelton, K. W.; Takeda, S.; Masamune, S. J. Chem Soc. D. 1971, 1516. Jones, R. R.; Bergman, R. G. J. Am. Chem. Soc. 1972, 94, 660.

(11) Myers, A. G. Tetrahedron Lett. 1987, 28, 4493. Myers, A. G.; Cohen, S. B.; Kwon, B.-M. J. Am. Chem. Soc. 1994, 116, 1670.

(12) Fujiwara, K.; Sakai, H.; Hirama, M. J. Org. Chem. 1991, 56, 1688.

(13) Myers, A. G.; Kuo, E. Y.; Finny, N. S. J. Am. Chem. Soc. 1989, 111, 8057. Nagata, R.; Yamanaka, H.; Murahashi, E.; Saito, I. Tetrahedron Lett. 1990, 31, 2907.

(14) Scheuplein, S. W.; Machinek, R.; Suffert, J.; Brückner, R. Tetrahedron Lett. 1993, 34, 6549.

(15) Stille, J. K.; Simpson, J. H. J. Am. Chem. Soc. 1987, 109, 2138.

(16) Hirama, M.; Fujiwara, K.; Shigematsu, K.; Fukazawa, Y. J. Am. Chem. Soc. 1989, 111, 4120.

(17) Fujiwara, K.; Kurisaki, A.; Hirama, M. Tetrahedron Lett. 1990, 31, 4329.

(18) Fujiwara, K.; Sakai, H.; Tanaka, T.; Hirama, M. Chem. Lett. 1994, 457.

(19) Tanaka, T.; Fujiwara, K.; Hirama, M. Tetrahedron Lett. 1990, 31, 5947.

(20) (a) Sugiyama, H.; Yamashita, K.; Fujiwara, T.; Saito, I. Tetrahedron 1994, 50, 1311. (b) Chin, D.-H.; Goldberg, I. H. J. Am. Chem. Soc. 1993, 115, 9341.

(21) Tokuda, M.; Fujiwara, K.; Hirama, M. Synlett 1991, 651.

(22) Hirama, M.; Gomibuchi, T.; Fujiwara, K.; Sugiura, Y.; Uesugi, M. J. Am. Chem. Soc. 1991, $113,9851$.

(23) Tokuda, M.; Fujiwara, K.; Gomibuchi, T.; Hirama, M.; Uesugi, M.; Sugiura, Y. Tetrahedron Lett. 1993, 34, 669.

(24) Galat, A.; Goldberg, I. H. Nucleic Acids Res. 1990, 18, 2093. Sugiyama, H.; Fujiwara, T.; Kawabata, H.; Yoda, N.; Hirayama, N.; Saito, I. J. Am. Chem. Soc. 1992, 114, 5573.

(25) (a) Ishiguro, M.; Imajo, S.; Hirama, M. J. Med. Chem. 1991, 34, 2366. (b) Tanaka, T.; Hirama, M.; Ueno, M.; Imajo, S.; Ishiguro, M.; Mizugaki, M.; Edo, K.; Komatsu, H. Tetrahedron Lett. 1991, 32, 3175. (c) Tanaka, T.; Hirama, M.; Fujita, K.; Imajo, S.; Ishiguro, M. J. Chem. Soc. Chem. Commun. 1993, 1205.

(26) Gomibuchi, T. unpublished results.

(27) Nishio, M.; Hirota, M. Tetrahedron 1989, 45, 7201.

(28) Kim, K.-H.; Kwon, B.-M.; Myers, A. G.; Rees, D. C. Science 1993, 262, 1042.

(29) Teplyakov, A.; Obmolova, G.; Wilson, K.; Kuromizu, K. Eur. J. Biochem. 1993, $213,737$.

(30) Imajo, S.; Ishiguro, M.; Tanaka, T.; Hirama, M.; Teplyakov, A. submitted for publication.

(31) (a) Takahashi, K.; Suzuki, T.; Hirama,, M. Tetrahedron Lett. 1992, 33, 4603. (b) Takahashi, K.; Tanaka, T.; Suzuki, T.; Hirama, M. Tetrahedron 1994, 50, 1327.

(32) (a) Konradsson, P.; Udodong, U.E.; Fraser-Reid, B. Tetrahedron Lett. 1990, 31, 4313. (b) Veeneman, G. H.; van Leeuwen, S. H.; van Boom, J. H. Tetrahedron Lett. 1990, 31, 1331.

(33) Gomibuchi, T.; Fujiwara, K.; Nehira, T.; Hirama, M. Tetrahedron Lett. 1993, 34, 5753. 